M. OKA

KODAI MATH. J.

12 (1989), 210-227

\title{
ON THE STRATIFICATION OF THE DISCRIMINANT VARIETIES
}

\author{
By Mutsuo OKA
}

\begin{abstract}
In this paper, we study the canonical stratification of the discriminant varieties of $A_{l}, B_{l}$ and $D_{l}$. We prove that this stratification enjoys strong geometric properties including the regularity.
\end{abstract}

\section{§1. Introduction.}

Let $R$ be a reduced irreducible root system in $\boldsymbol{R}^{l}$. Let $\mathscr{H}=\left\{H_{\alpha}\right\}(\alpha \in \Lambda)$ be the corresponding arrangement of the hyperplanes. The Weyl group $W$ is the group generated by the reflections along $\left\{H_{\alpha} ; \alpha \in \Lambda\right\}$. It acts on $C^{t}$ so that the quotient space $\boldsymbol{C}^{l} / W$ is isomorphic to the affine space $\boldsymbol{C}^{l}$ whose affine coordinate ring is the ring of the invariant polynomial $C\left[\xi_{1}, \cdots, \xi_{l}\right]^{W}$ (Chapter 6, [1]). Let $|\mathscr{H}|=\bigcup_{\alpha \in \Lambda} H_{\alpha}$. The action on the complement $\boldsymbol{C}^{l}-|\mathscr{H}|$ is free and $|\mathscr{H}|$ is $W$-invariant. We call the quotient space $|\mathscr{H}| / W$ the discriminant variety of the root system and we denote it by $\mathscr{D}$. The discriminant variety is a hypersurface in the quotient space $C^{l} / W$. There are many interesting results by many authors about the topology of the arrangement $|\mathscr{H}|$ or $C^{l+1}$ $|\mathscr{H}|$. See Orlik [6] and its references. The complement $C^{l}-\mathscr{D}$ is known to be a $K(\pi, 1)$-space by [2] and [3]. Let $\mathcal{S}$ be a stratification of $|\mathscr{H}|$ which is compatible with the $W$-action. For instance, we can take the minimal stratification $\mathcal{S}_{m \imath n}=\left\{H_{\varepsilon} ; \Xi \subset \Lambda\right\}$ where $H_{\varepsilon}=\bigcap_{\alpha \in Z} H_{\alpha}-\bigcup_{\alpha \notin Z} H_{\alpha}$. For a given $\mathcal{S}, \mathscr{D}$ inherits a canonical stratification $\overline{\mathcal{S}}$ which is defined by the images of the strata of $\mathcal{S}$. The purpose of this paper is to show that the discriminant variety for the arrangements of type $A_{l}, B_{l}$ and $D_{l}$ has canonical regular stratifications which are constructed in the above way. Here the regularity means the $b$ regularity in the sense of Whitney [7]. It is known that the $b$-regularity implies the $a$-regularity ([5]). For $A_{l+1}$ and $B_{l+1}$, we simply take $\mathcal{S}=\mathcal{S}_{m \imath n}$. As the stratification $\mathcal{S}$ for $D_{l+1}$, we take the restriction of $\mathcal{S}_{m i n}$ for $B_{l+1}$ to $D_{l+1}$.

Let $\subseteq$ be an analytic stratification of an analytic variety $V$ is an open set $U$ of $C^{n}$. Let $(M, N)$ be a pair of strata of $\mathscr{I}$ with $\bar{M} \supset N$ and let $q \in N$. Let $p(u)(0 \leqq u<1)$ be a real analytic curve such that $p(0)=q$ and $p(u) \in M$ for $u>0$.

Received October 14, 1988 
Let $T=\lim _{u \rightarrow 0} T_{p(u)} M$. We say that the pair $(M, N)$ has a unique tangential limit at $q$ if this limit $T$ depends only on $q$ and $M$. If $\mathscr{T}$ enjoys this property at any point $q$ of $N$ for any pair $(M ; N)$, we say that $\mathscr{T}$ has the unique tangential limits property. Of course, the existence of a stratification with the unique tangential limits property poses a strong geometric restriction on $V$.

We will show that the stratifications $\overline{\mathcal{S}}$ for $A_{l+1}$ and $B_{l+1}$-discriminants have the unique tangential limits property. For $D_{l+1}$-discriminant variety, this does not hold for certain pair of strata. However we will show that the limit of tangent spaces is at most one dimensional. The author would like to thank Professor P. Orlik and Professor K. Saito for the valuable informations and discussions.

\section{§ 2. $A_{l}$-arrangement.}

We first consider the $A_{l}$-arrangement. As a root system, $A_{l}$ is the restriction of $B_{l+1}$ to the following hyperplane

$$
L: \xi_{1}+\cdots+\xi_{l+1}=0 .
$$

The corresponding arrangement $\mathscr{H}$ consists of $\left(\begin{array}{c}l+1 \\ 2\end{array}\right)$ hyperplanes $\left\{\xi_{i}-\xi_{j}=0\right\}$ $(i<j)$ and the Weyl group $W$ is the symmetric group $S_{l+1}$. The invariant ring is generated by

$$
s_{\imath}=\sum_{\tau \in S_{l+1}} \xi_{\tau(1)} \cdots \xi_{\tau(i)} \quad(i=1, \cdots, l+1) .
$$

We refer to Chapter 6 of [1] for the basic results about the irreducible root systems. We use the following symmetric polynomials for the calculation's sake.

$$
\tau_{\imath}=\xi_{1}^{\imath}+\cdots+\xi_{l+1}^{\imath} \quad(i=1, \cdots, l+1) .
$$

Note that $\left\{\tau_{1}, \cdots, \tau_{l+1}\right\}$ is also a basis of the ring of invariant polynomials and that $s_{1}=\tau_{1}=0$ on $L$. We define the mapping $\Phi: C^{l+1} \rightarrow C^{l+1}$ by $\Phi\left(\xi_{1}, \cdots, \xi_{l+1}\right)=$ $\left(\tau_{1}, \cdots, \tau_{l+1}\right)$. Let $\bar{L}$ be the hyperplane in the quotient space defined by $\tau_{1}=0$. Let $\phi_{L}: L \rightarrow \bar{L}$ and $\phi:|\mathscr{H}| \rightarrow \mathscr{D}$ be the respective restriction of $\Phi$ to $L$ and $|\mathscr{H}|$. We have the following commutative diagrams.

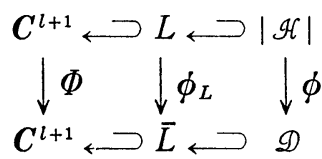

Here the horizontal maps are the respective inclusion maps. It is well-known that $\mathscr{D}$ is defined by $\Pi_{2<j}\left(\xi_{i}-\xi_{j}\right)^{2}=0$ which can be written in a weighted homogeneous polynomial of $\left\{s_{1}, \cdots, s_{l+1}\right\}$ or equivalently of $\left\{\tau_{1}, \cdots, \tau_{l+1}\right\}$. This is equal to the discriminant polynomial of $x^{l+1}-s_{1} x^{l}+\cdots+(-1)^{l+1} s_{l+1}=0$ in the 
usual sense ([4]).

Now we consider the stratification $S=S_{m \imath n}$ of $|\mathcal{H}|$. Let $\mathcal{C}_{1}$ be the set of the non-maximal subdivisions of the set $\{1, \cdots, l+1\}$. Namely an element $\mathscr{F}$ of $\mathcal{C}_{1}$ can be written as $\left\{I_{1}, \cdots, I_{k}\right\}$ w here $I_{i} \cap I_{j}=\emptyset$ for $i \neq j$ and $\bigcup_{j=1}^{k} I_{j}=\{1, \cdots$, $l+1\}$. The maximal element $\mathscr{M}=\{\{1\}, \cdots,\{l+1\}\}$ is excluded as $M(\mathscr{M})=$ $C^{l+1}-|\mathscr{H}|$. Note that the Weyl group $W$ acts canonically on $\mathcal{C}_{1}$. Let $\mathcal{C}_{2}$ be the set of the non-maximal partitions of the integer $l+1$. An element $\mathcal{K}$ of $\mathcal{C}_{2}$ is written as $\left\{m_{1}, \cdots, m_{k}\right\}$ such that $\sum_{j=1}^{k} m_{j}=l+1$ with $m_{j}>0$. For a subset $I$ of $\{1, \cdots, l+1\}$, we denote its cardinality by $|I|$. Then there is a canonical surjection from $\mathcal{C}_{1}$ to $\mathcal{C}_{2}$ by $\mathscr{I} \mapsto|I|$ where $|\mathscr{I}|=\left\{\left|I_{1}\right|, \cdots,\left|I_{k}\right|\right\}$. For each $\mathscr{F}=\left\{I_{1}, \cdots, I_{k}\right\}$ of $\mathcal{C}_{1}$, we define

$$
M(\mathscr{I})=\left\{\xi=\left(\xi_{i}\right) \in C^{l+1} ; \xi_{i}=\xi, \Longleftrightarrow \exists a ;\{i, j\} \subset I_{a}\right\} .
$$

It is clear that $\{M(\mathscr{I})\}_{\mathscr{I} \in \mathcal{C}_{1}}$ is equal to $\mathcal{S}=\mathcal{S}_{m \imath n}$ which is a regular stratification of $|\mathscr{H}|$. Let $\mathscr{I}=\left\{I_{1}, \cdots, I_{k}\right\}$ and $\mathcal{G}=\left\{J_{1}, \cdots, J_{m}\right\}$ be elements of $\mathcal{C}_{1}$. $\mathscr{I}$ is called a subdivision of $\mathcal{G}$ if for each $i$, there exists a $j$ such that $I_{i} \subset J_{j}$. We define a partial ordering in $\mathcal{C}_{1}$ (respectively in $\mathcal{C}_{2}$ ) by $\mathscr{F}>\mathcal{G}$ if and only if $\mathscr{F}$ is a subdivision of $\mathcal{G}$. (Respectively $|\mathscr{F}| \succ|\mathcal{G}| \Leftrightarrow|\mathscr{F}|$ is a subpartition of $|\mathcal{G}|$.) The canonical map $\mathscr{F} \mapsto|\mathscr{F}|$ is obviously order-preserving.

Proposition (2.5). Let $\mathscr{T}, \mathscr{F}^{\prime} \in \mathcal{C}_{1}$. The following conditions are equivalent.

(i) $\overline{M(\mathscr{I})} \supseteqq M\left(\mathscr{I}^{\prime}\right)$. (ii) $\overline{M(\mathscr{F})} \cap M\left(\mathscr{F}^{\prime}\right) \neq \emptyset$. (iii) $\mathscr{F} \succ \mathscr{I}^{\prime}$.

Proposition (2.6). Let $\mathscr{F}, \mathscr{F}^{\prime} \in \mathcal{C}_{1}$. (I) The following conditions are equivalent.

(i ) $\phi(M(\mathscr{F}))=\phi\left(M\left(\mathscr{F}^{\prime}\right)\right)$. (ii) $\phi(M(\mathscr{F})) \cap \phi\left(M\left(\mathscr{F}^{\prime}\right)\right)=\emptyset$.

(iii) There exists an element $g \in W$ such that $g(M(\mathscr{F}))=M\left(\mathscr{F}^{\prime}\right)$.

(iv) $|\mathscr{F}|=\left|\mathscr{F}^{\prime}\right|$ in $\mathcal{C}_{2}$.

(II) $\overline{\phi(M(\mathscr{F}))} \supseteqq \phi\left(M\left(\mathscr{F}^{\prime}\right)\right)$ if and only if $|\mathscr{F}|>\left|\mathscr{F}^{\prime}\right|$.

Proof. Proposition (2.5) is immediate from the definition of $M(\mathscr{F})$. We prove Proposition (2.6). The equivalence (iii) $\Leftrightarrow$ (iv) is obvious. The implications (iii) $\Rightarrow($ i $) \Rightarrow$ (ii) are also trivial. Assume that $\phi(\xi)=\phi\left(\xi^{\prime}\right)$ for some $\xi \in M(\mathscr{I})$ and $\xi^{\prime} \in M\left(\mathscr{F}^{\prime}\right)$. This implies that there exists a $g \in W$ such that $g(\xi)=\xi^{\prime}$. As $\mathscr{H}$ is invariant by the action of $W$, we can write $g(M(\mathscr{F}))=M(\mathcal{G})$ for some $\mathscr{G} \in \mathcal{C}_{1}$. As $\{M(\mathscr{I})\}_{\mathscr{F} \in C_{1}}$ are disjoint, this implies $\mathscr{I}^{\prime}=\mathcal{G}$. Thus (ii) $\Rightarrow$ (iii). As $\overline{\phi(M(\mathscr{I}))}=$ $\overline{\phi(M(\mathscr{F}))}$, the assertion (II) is an immediate consequence of (I) and Proposition (2.5).

Definition (2.7). For $\mathcal{K} \in \mathcal{C}_{2}$, we define $V(\mathcal{K})=\phi(M(\mathscr{I}))$ where $|\mathscr{I}|=\mathcal{K}$.

We define an important vector-valued function $X(x)$ by

$$
X(x)=\left(x, x^{2}, \cdots, x^{l+1}\right) .
$$


Let $X^{\prime}(x)=\left(1,2 x, \cdots,(l+1) x^{l}\right)$ be the derivative of $X(x)$. Then $\Phi(\xi)=\sum_{\imath=1}^{l+1} X\left(\xi_{i}\right)$ and the tangential map $d \Phi_{\xi}: T_{\xi} C^{l+1} \rightarrow T_{\Phi(\xi)} C^{l+1}$ satisfies $d \Phi_{\xi}\left(\frac{\partial}{\partial \xi_{i}}\right)=\sum_{\jmath=1}^{l+1} j \xi^{\jmath-1} \frac{\partial}{\partial \tau_{\jmath}}$. We identify the tangent space $T_{\Phi(\xi)} \boldsymbol{C}^{l+1}$ with $\boldsymbol{C}^{l+1}$ in a canonical way. Then the above equality says

$$
d \Phi_{\xi}\left(\frac{\partial}{\partial \xi_{i}}\right)=X^{\prime}\left(\xi_{i}\right), \quad i=1, \cdots, l+1 .
$$

For any subset $I$ of $\{1, \cdots, l+1\}$, we define

$$
\frac{\partial}{\partial \xi_{I}}=\frac{1}{|I|} \sum_{i \in I} \frac{\partial}{\partial \xi_{i}}, \quad \xi_{I}=\frac{1}{|I|} \sum_{i \in I} \xi_{i} .
$$

Let $\mathscr{F}=\left\{I_{1}, \cdots, I_{k}\right\}$ and let $\xi \in M(\mathscr{I})$. As $\xi$, does not depend on $\jmath \in I_{\imath}$ for $i$ being fixed, we have $\xi_{\jmath}=\xi_{I_{\imath}}$ for any $j \in I_{\imath}$.

Proposition (2.11). Let $\mathscr{F}=\left\{I_{1}, \cdots, I_{k}\right\}$ and let $\xi \in M(\mathscr{F})$.

(i) $T_{\xi} M(\mathscr{I})$ is the (k-1)-dimensional vector space which is equal to

$$
T_{\xi} M(\mathscr{F})=\left\{\sum_{t=1}^{k} \lambda_{t} \frac{\partial}{\partial \xi_{I_{t}}} ; \sum_{t=1}^{k} \lambda_{t}=0\right\} .
$$

(ii) The restriction $\phi: M(\mathscr{F}) \rightarrow V(|\mathscr{F}|)$ is a finite covering.

(iii) $V(|\mathscr{I}|)$ is non-singular and

$$
T_{\phi(\xi)} V(|\mathscr{F}|)=\left\{\sum_{t=1}^{k} \lambda_{t} X^{\prime}\left(\xi_{I_{t}}\right) ; \sum_{t=1}^{k} \lambda_{t}=0\right\} .
$$

Proof. (i) is obvious by the definition of $M(\mathscr{I})$. Thus

$$
d \Phi_{\xi}\left(T_{\xi} M(\mathscr{F})\right)=\left\{\sum_{t=1}^{k} \lambda_{t} X^{\prime}\left(\xi_{I_{t}}\right) ; \sum_{t=1}^{k} \lambda_{t}=0\right\} .
$$

By the Vandermonde determinant formula, this image has dimension $(k-1)$. Thus the restriction $\phi \mid M(\mathscr{F})$ is a submersion and the local image by $\phi$ is smooth. Now assume that $\phi(\xi)=\phi(\eta)$ for $\xi, \eta \in M(\mathscr{F})$ with $\xi \neq \eta$. Then there exists a permutation $g \in S_{l+1}$ so that $g(\xi)=\eta$. Then $g(M(\mathscr{F}))=M(\mathscr{F})$. Thus the local images near $\xi$ and $\eta$ by $\phi$ coincide. This proves that $V(|\mathscr{F}|)$ is smooth and the assertions (ii) and (iii) follow immediately.

Let us examine the order of the covering $\phi: M(\mathscr{F}) \rightarrow V(|\mathscr{F}|)$ more explicitly. Let $\left\{\alpha_{1}, \cdots, \alpha_{m}\right\}=\left\{n: \exists i, n=\left|I_{\imath}\right|\right\}$. Clearly we have $m \leqq k$ and $\left\{\alpha_{2}\right\}$ are mutually distinct. Let $\rho_{i}$ be the number of $j$ 's such that $\left|I_{j}\right|=\alpha_{\imath}(i=1, \cdots, k)$. We consider the subgroups

$$
W(\mathscr{F})=\{g \in W ; g(M(\mathscr{F}))=M(\mathscr{F})\}, \quad I(\mathscr{F})=\{g \in W ; g \mid M(\mathscr{F})=i d\} .
$$

Then $I(\mathscr{I})$ is a normal subgroup of $W(\mathscr{F})$ and the quotient group $W(\mathscr{I}) / I(\mathscr{I})$ acts freely on $M(\mathscr{F})$ with the quotient space $V(|\mathscr{F}|)$. More precisely let $\bar{g} \in W(\mathscr{F}) / I(\mathscr{I})$. Then for each $s=1, \cdots, m, \bar{g}$ induces a permutation of 
$\left\{\xi_{I} ;\left|I_{j}\right|=\alpha_{s}\right\}$. Thus we have

PROPOSITION (2.12). There is a canonical isomorphism $W(\mathscr{I}) / I(\mathscr{I}) \cong S_{\rho_{1}} \times \cdots$ $\times S_{\rho_{m}}$. Thus the order of the above covering is $\rho_{1} ! \cdots \rho_{m} !$.

Let $f(x)$ be a vector valued rational function of one variable. We define the rational functions $f_{k}\left(x_{1}, \cdots, x_{k}\right)(k=1, \cdots, l+1)$ inductively by $f_{1}\left(x_{1}\right)=f\left(x_{1}\right)$ and

$$
\begin{aligned}
& f_{k}\left(x_{1}, \cdots, x_{k}\right) \\
& \quad=\left\{f_{k-1}\left(x_{1}, \cdots, x_{k-2}, x_{k-1}\right)-f_{k-1}\left(x_{1}, \cdots, x_{k-2}, x_{k}\right)\right\} /\left(x_{k-1}-x_{k}\right)
\end{aligned}
$$

We call $f_{k}\left(x_{1}, \cdots, x_{k}\right)$ the $k$-fold derived function of $f(x)$.

Proposition (2.14). We have the following formulae.

(i) $f\left(x_{k}\right)=f\left(x_{1}\right)+\sum_{j=2}^{k}\left(\prod_{h=1}^{j-1}\left(x_{k}-x_{h}\right)\right) f_{j}\left(x_{1}, \cdots, x_{j}\right)$

(ii) $f_{s+1}\left(x_{1}, \cdots, x_{s}, x_{s+k}\right)$

$$
=f_{s+1}\left(x_{1}, \cdots, x_{s+1}\right)+\sum_{j=2}^{k}\left(\prod_{h=1}^{j-1}\left(x_{s+k}-x_{s+h}\right) f_{s+j}\left(x_{1}, \cdots, x_{s+j}\right) .\right.
$$

Proof. As (i) is a special case of (ii), we prove (ii) by the induction on $k$. The assertion on $k=1$ is trivial. We assume the assertion for $k-1$. By the definition of the derived function, we have

$$
\begin{aligned}
f_{s+1}\left(x_{1}, \cdots, x_{s}, x_{s+k}\right)-f_{s+1}\left(x_{1}, \cdots, x_{s}, x_{s+1}\right)=\left(x_{s+k}-x_{s+1}\right) f_{s+2}\left(x_{1}, \cdots, x_{s+1}, x_{s+k}\right) \\
=\left(x_{s+k}-x_{s+1}\right) f_{s+2}\left(x_{1}, \cdots, x_{s+2}\right) \\
\quad+\left(x_{s+k}-x_{s+1}\right) \sum_{j=2}^{k}\left(\prod_{h=1}^{j-1}\left(x_{s+k}-x_{s+1+h}\right)\right) f_{s+1+j}\left(x_{1}, \cdots, x_{s+1+j}\right) \\
=\sum_{j=2}^{k}\left(\prod_{h=1}^{j-1}\left(x_{s+k}-x_{s+h}\right)\right) f_{s+j}\left(x_{1}, \cdots, x_{s+j}\right) .
\end{aligned}
$$

This completes the proof.

Now we consider the derived functions $X_{k}\left(x_{1}, \cdots, x_{k}\right)$ and $X_{k}^{\prime}\left(x_{1}, \cdots, x_{k}\right)$ of $X(x)$ and $X^{\prime}(x)$ respectively. The following Lemma plays an important role throughout this paper.

LEMMA (2.15). Let $a_{k, j}$ and $b_{k, j}$ be the $j$-th coordinate of $X_{k}\left(x_{1}, \cdots, x_{k}\right)$ and $X_{k}^{\prime}\left(x_{1}, \cdots, x_{k}\right)$ respectively. Then $a_{k, \jmath}, b_{k, j}$ are symmetric polynomials of $x_{1}, \cdots, x_{k}$ defined by

(i) $a_{k, k+\jmath}=\sum_{\nu_{1}+\cdots+\nu_{k}=j+1} x_{1}^{\nu_{1} \cdots x_{k}^{\nu} k}, \quad b_{k, k+\jmath}=(k+j)_{\nu_{1}+\cdots+\nu_{k}=j} x_{1}^{\nu_{1}} \cdots x_{k}^{\nu k}$

(ii) $X_{k}(x, \cdots, x)=X^{(k-1)}(x) /(k-1) !, \quad X_{k}^{\prime}(x, \cdots, x)=X^{(k)}(x) /(k-1)$ ! 
where $X^{(j)}(x)=\left(\frac{d}{d x}\right)^{\jmath} X(x)$

Proof. (i) is immediate from the inductive calculation and the equality: $\left(x^{a}-y^{a}\right) /(x-y)=x^{a-1}+x^{a-2} y+\cdots+y^{a-1}$. The assertion (ii) follows immediately from (i).

LeMmA (2.16). Let $\xi \in M(\mathscr{F})$ and let $\mathscr{F}=\left\{I_{1}, \cdots, I_{k}\right\}$. Then

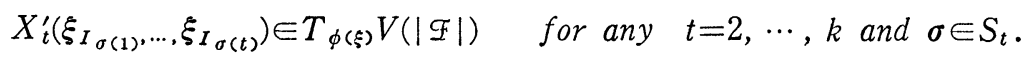

Proof. By Proposition (2.11), we have that

$$
X^{\prime}\left(\xi_{I_{i}}\right)-X^{\prime}\left(\xi_{I_{j}}\right)=\left(\xi_{I_{\imath}}-\xi_{I_{j}}\right) X_{2}^{\prime}\left(\xi_{I_{i}}, \xi_{I_{j}}\right) \in T_{\phi(\xi)} V(|\mathscr{I}|) \quad(i \neq j) .
$$

This implies that $X_{2}^{\prime}\left(\xi_{I_{i}}, \xi_{I_{j}}\right) \in T_{\phi(\xi)} V(|\mathscr{F}|)$ for $i \neq j$. Now the assertion follows by an easy inductive argument.

The following is a generalization of the Vandermonde determinant formula and it plays a key role to show the linear independence of certain vectors in the later arguments.

LEMMA (2.17). (Generalized Vandermonde formula) Let $\lambda_{1}, \cdots, \lambda_{k}$ be mutually distinct complex numbers and let $\Re=\left\{\nu_{1}, \cdots, \nu_{k}\right\}$ be an element of $\mathcal{C}_{2}$. Then we have the formula:

$$
\operatorname{det}\left({ }^{t} X^{\prime}\left(\lambda_{1}\right), \cdots,{ }^{t} X^{\left(\nu_{1}\right)}\left(\lambda_{1}\right), \cdots,{ }^{t} X^{\prime}\left(\lambda_{k}\right), \cdots,{ }^{t} X^{\left(\nu_{k}\right)}\left(\lambda_{k}\right)\right)=(l+1) ! \prod_{j>2}\left(\lambda_{j}-\lambda_{2}\right)^{\nu_{\imath} \nu^{\nu}} .
$$

In particular, $\left\{X^{(j)}\left(\lambda_{2}\right)\right\}\left(j=1, \cdots, \nu_{i}, i=1, \cdots, k\right)$ are linearly independent.

Proof. Let $\Psi\left(x_{1}, \cdots, x_{l+1}\right)=\operatorname{det}\left({ }^{t} X^{\prime}\left(x_{1}\right), \cdots,{ }^{t} X^{\prime}\left(x_{l+1}\right)\right)$. Then it is easy to see that

$$
\Psi\left(x_{1}, \cdots, x_{l+1}\right)=(l+1) ! \prod_{j>\imath}\left(x_{j}-x_{\imath}\right)
$$

by the Vandermonde determinant formula. We consider the differential operators :

$$
D_{i}=\left(\frac{\partial}{\partial x_{\nu_{1}+\cdots+\nu_{i-1}+2}}\right)^{1} \cdots\left(\frac{\partial}{\partial x_{\nu_{1}+\cdots+\nu_{i}}}\right)^{\nu_{i}-1} \quad \text { and } \quad D=D_{1} \cdots D_{k} .
$$

Let $E=\left\{(j, h) ; \nu_{1}+\cdots+\nu_{i-1}+1 \leqq h<j \leqq \nu_{1}+\cdots+\nu_{i}, i=1, \cdots, k\right\}$ and let $\mathcal{E}$ be the ideal generated by $\left\{x_{j}-x_{h} ;(j, h) \in E\right\}$. As $\sum_{j=1}^{\nu_{i}^{i}-1} j=\left(\begin{array}{c}\nu_{i} \\ 2\end{array}\right)$, it is easy to see that

$$
D \Psi \equiv(l+1) ! \prod_{(\jmath, h) \notin E}\left(x_{j}-x_{h}\right) \text { modulo } \mathcal{E} .
$$

Thus the assertion follows immediately from 


$$
\begin{aligned}
& \operatorname{det}\left({ }^{t} X^{\prime}\left(\lambda_{1}\right), \cdots,{ }^{t} X^{\left(\nu_{1}\right)}\left(\lambda_{1}\right), \cdots,{ }^{t} X^{\prime}\left(\lambda_{k}\right), \cdots,{ }^{t} X^{\left(\nu_{k}\right)}\left(\lambda_{k}\right)\right) \\
& =(D \Psi)(\underbrace{\lambda_{1}, \cdots, \lambda_{1}}_{\nu_{1}}, \cdots, \underbrace{\lambda_{k}, \cdots, \lambda_{k}}_{\nu_{k}})=(l+1) ! \prod_{j>2}\left(\lambda_{j}-\lambda_{2}\right)^{\nu^{\nu^{\nu} j} .}
\end{aligned}
$$

Here the last equality is due to (2.19).

\section{§3. Regularity and the limit of the tangent space.}

Now we are ready to show the regularity of the stratification $\overline{\mathcal{S}}$ of the discriminant variety of $A_{l+1}$-arrangement and the unique tangential limits property. Let $M(\mathscr{I})$ and $M(\mathcal{G})$ be stratum of $\mathcal{S}$ such that $\overline{M(\mathscr{T})} \supset M(\mathcal{G})$. Let $q$ be an arbitrary point of the stratum $V(|\mathcal{Q}|)$ and let $\bar{p}(u)$ and $\bar{q}(u)$ be real analytic curves defined on the interval $[0,1]$ such that (i) $\bar{p}(0)=\bar{q}(0)=q$ and $\bar{q}(u) \in V(|\mathcal{Q}|)$ for any $u \in[0,1]$. (ii) $\bar{p}(u) \in V(|\mathscr{F}|)$ for $u>0$. We also assume that

$$
\lim _{u \rightarrow 0} T_{\bar{p}(u)} V(|\mathscr{F}|)=T, \quad \lim _{u \rightarrow 0}[\bar{p}(u), \bar{q}(u)]=\gamma .
$$

Here $[\bar{p}(u), \bar{q}(u)]$ is the line spanned by $\bar{p}(u)-\bar{q}(u)$. Changing the parameter $u$ by $u^{1 / m}$ for some integer $m$ if necessary, we may assume that there are lifting real analytic curves $p(u)$ and $q(u)$ in $\overline{M(\mathscr{I})}$ and $M(\mathcal{G})$ respectively so that $\bar{p}(u)=\phi(\phi(u))$ and $\bar{q}(u)=\phi(q(u))$ respectively. We may assume that $p(0)=q(0)$ and let $\eta=p(0) \in M(\mathcal{G})$. Let $\mathcal{G}=\left\{J_{1}, \cdots, J_{m}\right\}$. By Proposition (2.5), we can write $\mathscr{I}=\left\{J_{i, j} ; i=1, \cdots, m, i=1, \cdots, \nu_{i}\right\}$ where $J_{i, j} \subset J_{i}$ for $j=1, \cdots, \nu_{i}$.

THEOREM (3.2). $\quad \overline{\mathcal{S}}$ is a regular stratification with the unique tangential limits property. Namely (i) $T$ is generated by

$$
\left\{\sum_{i=1}^{m} \lambda_{\imath} X^{\prime}\left(\eta_{J_{i}}\right) ; \sum_{i=1}^{m} \lambda_{2}=0\right\} \bigcup\left\{X^{(j)}\left(\eta_{J_{\imath}}\right), 1 \leqq i \leqq m, 2 \leqq \jmath \leqq \nu_{i}\right\} .
$$

(ii) (Regularity) $\gamma \in T$.

Proof. By Proposition (2.11), the vectors $\lambda_{1} X^{\prime}\left(p(u)_{J_{1,1}}\right)+\cdots+\lambda_{m} X^{\prime}\left(p(u)_{J_{m, 1}}\right)$ with $\sum_{\imath=1}^{m} \lambda_{2}=0$ are contained in $T_{\bar{p}(u)} V(|\mathscr{F}|)$. Thus by taking the limit as $u \rightarrow 0$, we see that $\sum_{\imath=1}^{m} \lambda_{2} X^{\prime}\left(\eta_{J_{i}}\right) \in T$. This gives only a subspace of $T$ of dimension $m-1$. We still need $\nu_{1}+\cdots+\nu_{m}-m$ independent vectors to generate $T$. For this purpose, we apply Lemma (2.15). We know that $X_{k}^{\prime}\left(p(u)_{J_{\imath}, 1}, \cdots\right.$, $\left.p(u)_{J_{\imath, k}}\right) \in T_{\bar{p}(u)} V(|\mathscr{F}|)\left(2 \leqq k \leqq \nu_{i}, 1 \leqq i \leqq m\right)$. We take the limits of these vectors as $u \rightarrow 0$ and we apply Lemma (2.15) to obtain that $X^{(j)}\left(\eta_{J_{i}}\right) \in T\left(2 \leqq j \leqq \nu_{i}\right.$, $1 \leqq i \leqq m)$. Now we apply Lemma (2.17) to see that the vectors $\left\{X^{(j)}\left(\eta_{J_{i}}\right)\right.$; $\left.1 \leqq i \leqq m, 1 \leqq j \leqq \nu_{i}\right\}$ are linearly independent. This completes the proof of (i).

Now we consider the regularity (ii). Using the equality $\sum_{j=1}^{\nu_{i}}\left|J_{\imath, j}\right|=\left|J_{\imath}\right|$, we have 


$$
\bar{p}(u)-\bar{q}(u)=\sum_{i=1}^{m} \sum_{j=1}^{\nu_{i}}\left|J_{i, j}\right|\left(X\left(p(u)_{J_{\imath, j}}\right)-X\left(q(u)_{J_{i}}\right)\right) .
$$

Using Proposition (2.14), we can write

$$
X\left(p(u)_{J_{i, j}}\right)-X\left(q(u)_{J_{i}}\right)=\sum_{h=1}^{j} \alpha_{i, j, h}(u) X_{h+1}\left(q(u)_{J_{i}}, p(u)_{J_{i, 1}}, \cdots, p(u)_{J_{i, h}}\right)
$$

where $\alpha_{\imath, \jmath, h}(u)$ is defined by

$$
\alpha_{\imath, \jmath, h}(u)=\left(p(u)_{J_{i}, j}-q(u)_{J_{i}}\right) \prod_{k=1}^{h-1}\left(p(u)_{J_{i}, j}-p(u)_{J_{i, k}}\right), \quad h=1, \cdots, \nu_{i} .
$$

Substituting (3.4) in (3.3), we obtain

$$
\bar{p}(u)-\bar{q}(u)=\sum_{i=1}^{m} \sum_{h=1}^{\nu_{i}} \alpha_{\imath, h}(u) X_{h+1}\left(q(u)_{J_{i}}, p(u)_{J_{\imath, 1},}, \cdots, p(u)_{J_{\imath, h}}\right) .
$$

where $\alpha_{\imath, h}(u)=\sum_{j=h}^{\nu}\left|J_{\imath, j}\right| \alpha_{\imath, \jmath, h}(u)$. In particular, we have

$$
\alpha_{\imath, 1}(u)=\sum_{j=1}^{\nu_{i}}\left|J_{\imath, j}\right|\left(p(u)_{J_{i, j}}-q(u)_{J_{i}}\right) .
$$

We define a non-negative integer $\beta$ by

$$
\beta=\min \left\{\operatorname{order}\left(\alpha_{\imath, h}(u)\right) ; i=1, \cdots, m, h=1, \cdots, \nu_{i}\right\}
$$

and let $\alpha_{\imath, h}(u)=\alpha_{\imath, h} u^{\beta}+$ (higher terms). Then (3.6) and Lemma (2.15) imply that

$$
\bar{p}(u)-\bar{q}(u)=\left(\sum_{i=1}^{m} \sum_{h=1}^{\nu_{i}} \alpha_{\imath, h} X^{(h)}\left(\eta_{J_{i}}\right) / h !\right) u^{\beta}+\text { (higher terms). }
$$

By the Generalized Vandermonde formula (Lemma (2.17)), we can see easily that

$$
\sum_{i=1}^{m} \sum_{h=1}^{\nu_{i}} \alpha_{\imath, h} X^{(h)}\left(\eta_{J_{i}}\right) / h ! \neq 0 \text { and } \gamma=\left[\sum_{i=1}^{m} \sum_{h=1}^{\nu_{i}} \alpha_{\imath, h} X^{(h)}\left(\eta_{J_{i}}\right) / h !\right] .
$$

Here $[v]$ denotes the line generated by the vector $v$. Thus the assertion (ii) of Theorem (3.2) follows immediately from (i) and (3.10) and the following.

$$
\text { ASSERTION (3.11). } \quad \sum_{i=1}^{m} \alpha_{\imath, 1}=0 \text {. }
$$

Proof. By (3.7) we have

$$
\sum_{i=1}^{m} \alpha_{\imath, 1}(u)=\sum_{i=1}^{m} \alpha_{\imath, 1} t^{\beta}+(\text { higher terms })=\sum_{i=1}^{m} \sum_{j=1}^{\nu}\left|J_{\imath, j}\right| p(u)_{J_{i}, j}-\sum_{i=1}^{m}\left|J_{i}\right| q(u)_{J_{i}} \equiv 0 .
$$

The last equality is derived from the fact that $p(u)$ and $q(u)$ are in the hyperplane $L$. Now the assertion is immediate from the above equality. 


\section{§4. $B_{l+1}$-arrangement.}

Let $R$ be the root system of type $B_{l+1}$ in $\boldsymbol{R}^{l+1}$. The corresponding arrangement $\mathcal{H}$ consists of $2\left(\begin{array}{c}l+1 \\ 2\end{array}\right)+l+1$ hyperplanes: $\left\{\xi_{i} \pm \xi_{j}=0\right\}$ and $\left\{\xi_{i}=0\right\}$. The Weyl group $W$ is isomorphic to a semi-direct product of the symmetric group $S_{l+1}$ and the abelian group $(\boldsymbol{Z} / 2 \boldsymbol{Z})^{l+1}$ (Chapter $\left.6,[1]\right)$. The invariant polynomial ring is generated by

$$
t_{\imath}=\sum_{\tau \in S_{l+1}} \xi_{\tau(1)}^{2} \cdots \xi_{\tau(i)}^{2}, \quad i=1, \cdots, l+1 .
$$

We will use the following generators.

$$
\zeta_{i}=\xi_{1}^{22}+\cdots+\xi_{l+1}^{2 i} \quad i=1, \cdots, l+1 .
$$

Let $\Phi: \boldsymbol{C}^{l+1} \rightarrow \boldsymbol{C}^{l+1} / W \cong \boldsymbol{C}^{l+1}$ be the map defined by $\boldsymbol{\xi} \mapsto\left(\boldsymbol{\zeta}_{1}(\xi), \cdots, \boldsymbol{\zeta}_{l+1}(\xi)\right)$. We take $\mathcal{S}=\mathcal{S}_{m \imath n}$. The stratification $\mathcal{S}$ can be described as follows. Let $\mathcal{E}_{1}$ be the set of the subdivisions of the non-empty subsets of $\{1, \cdots, l+1\}$. Namely an element $\mathscr{F} \in \mathcal{E}_{1}$ can be written as $\mathscr{F}=\left\{I_{1}, \cdots, I_{k}\right\}$ where each $I_{2}$ is non-empty and $I_{i} \cap I_{j}=\emptyset$ for $i \neq j$. Let $\mathcal{S}(\mathscr{F})=\bigcup_{i=1}^{k} I_{i}$ and $\mathscr{F}^{c}=\{1, \cdots, l+1\}-S(\mathscr{F})$. Let $\mathcal{E}_{2}$ be the set of the partitions of the integer $m$ for $m=1, \cdots, l+1$. There is a canonical surjective mapping from $\mathcal{E}_{1}$ to $\mathcal{E}_{2}$ by $\mathscr{F} \mapsto|\mathscr{F}|=\left\{\left|I_{1}\right|, \cdots,\left|I_{k}\right|\right\}$. Let

$$
M(\mathscr{F})=\left\{\boldsymbol{\xi} \in \boldsymbol{C}^{l+1} \text {; (i) } \xi_{i}=0 \Leftrightarrow i \in \mathscr{F}^{c} \text {, (ii) } \xi_{i}^{2}=\xi_{j}^{2} \Leftrightarrow\{i, j\} \cong \exists I_{s}\right\}
$$

We omit $\mathscr{M}=\{\{1\}, \cdots,\{l+1\}\}$ and $|\mathscr{M}|$ from $\mathcal{E}_{1}$ and $\mathcal{E}_{2}$ respectively as $M(\mathscr{M})$ and $V(|\mathscr{M}|)$ are nothing but the complement $C^{l+1}-|\mathscr{H}|$ and $C^{l+1}-\mathscr{D}$. Let $\alpha=\sum_{i=1}^{k}\left|I_{i}\right|-k$. Then $M(\mathscr{F})$ is a disjoint union of $2^{\alpha}$ connected components corresponding th sign of $\xi_{i}= \pm \xi$, in the definition of $M(\mathscr{I})$. But they are in the same $W$-orbit. (Recall that the reflection along $\left\{\xi_{i}=0\right\}$ is the multiplication by -1 in the $i$-th coordinate.) Thus each connected component is mapped by $\phi$ onto the same stratum of $\overline{\mathcal{S}}$. We define partial orderings in $\mathcal{E}_{1}$ and $\mathcal{E}_{2}$ as follows. Let $\mathscr{F}=\left\{I_{1}, \cdots, I_{k}\right\}$ and $\mathcal{G}=\left\{J_{1}, \cdots, J_{n}\right\} . \mathscr{F}>\mathcal{G}$ if and only if (i) $\mathscr{F}^{c} \cong \mathcal{G}^{c}$, (ii) $\tilde{\mathscr{F}} \succ \tilde{G}$ in $\mathcal{C}_{1}$. Here $\tilde{\mathscr{F}}$ is defined by $\left\{\mathscr{F}^{c}, I_{1}, \cdots, I_{k}\right\} \in \mathcal{C}_{1}$. Similarly we define $|\mathscr{F}|>|\mathcal{G}|$ if and only if (i) $\left|\mathscr{F}^{c}\right| \leqq\left|\mathscr{G}^{c}\right|$, (ii) $|\tilde{\mathscr{F}}|>|\tilde{G}|$ in $\mathcal{C}_{2}$. Now the following propositions are completely parallel to Proposition (2.5) and Proposition (2.6).

Proposition (4.3). Let $\mathscr{F}, \mathcal{G} \in \mathcal{E}_{1}$. The following conditions are equivalent.
(i) $\overline{M(\mathscr{I})} \supseteqq M(\mathcal{G})$.
(ii) $\overline{M(\mathscr{I})} \cap M(\mathcal{Q}) \neq \emptyset$.
(iii) $\mathscr{F}>\mathcal{G}$.

PROPOSITION (4.4). Let $\mathscr{I}, \mathcal{G} \in \mathcal{E}_{1}$. The following conditions are equivalent. (i) $\phi(M(\mathscr{I}))=\phi(M(\mathcal{G}))$. (ii) There exists a $g \in W$ such that $g(M(\mathscr{I}))=M(\mathcal{G})$. (iii) $|\mathscr{F}|=|\mathcal{Q}|$. 
Thus for a $\mathcal{K} \in \mathcal{E}_{2}$ we can define $V(\mathcal{K})=\phi(M(\mathscr{F}))$ for any $\mathscr{F} \in \mathcal{E}_{1}$ such that $|\mathscr{F}|=\mathcal{K}$. Now we study the tangential map. Note that

$$
d \Phi_{\xi}\left(\frac{\partial}{\partial \xi_{i}}\right)=2 \xi_{i} X^{\prime}\left(\xi_{i}^{2}\right) .
$$

For each $I \subset\{1, \cdots, l+1\}$, we define $m(I)=\min \{i ; i \in I\} . \quad$ Let $\mathscr{F}=\left\{I_{1}, \cdots, I_{k}\right\} \in \mathcal{E}_{1}$ and let $\xi \in \mathscr{F}$. We define $\tilde{\xi} \in M(\mathscr{F})$ by

$$
\tilde{\xi}_{j}= \begin{cases}\xi_{m\left(I_{i}\right)} & \text { if } \quad j \in I_{i} \\ 0 & \text { if } j \in \mathscr{F}^{c} .\end{cases}
$$

It is easy to see that $\tilde{\xi}$ is in the $W$-orbit of $\xi$. We also define

$$
\frac{\widetilde{\partial}}{\partial \xi_{I_{2}}}=\frac{1}{\left|I_{\imath}\right|} \sum_{j \in I_{i}}\left(\xi_{j} / \xi_{m\left(I_{2}\right)}\right) \frac{\partial}{\partial \xi_{j}} \text {. }
$$

Note that $\xi_{j} / \xi_{m\left(I_{i}\right)}= \pm 1$ and $\xi_{j}^{2}=\xi_{m\left(I_{i}\right)}^{2}=\tilde{\xi}_{I_{\imath}}^{2}$ for each $j \in I_{\imath}$. It is easy to see that $\frac{\widetilde{\partial}}{\partial \xi_{I_{2}}} \in T_{\xi} M(\mathscr{F})$ and $d \Phi_{\xi}\left(\frac{\widetilde{\partial}}{\partial \xi_{I_{i}}}\right)=2 \tilde{\xi}_{I_{i}} X^{\prime}\left(\tilde{\xi}_{I_{i}}^{2}\right)$. Now Proposition (2.11) and Lemma (2.15) can be translated into the following form.

PROPOSITION (4.7). Let $\mathscr{I}=\left\{I_{1}, \cdots, I_{k}\right\} \in \mathcal{E}_{1}$. Then

(i) The dimension of $T_{\xi} M(\mathscr{F}) i$ is $k$ and it is generated by $\left\{\frac{\widetilde{\partial}}{\partial \xi_{I_{2}}} ; i=1, \cdots, k\right\}$.

(ii) The restriction $\phi: M(\mathscr{I}) \rightarrow V(|\mathscr{F}|)$ is a finite covering.

(iii) $V(|\mathscr{F}|)$ is non-singular and $T_{\phi(\xi)} V(|\mathscr{F}|)$ is generated by $\left\{X^{\prime}\left(\tilde{\xi}_{I_{i}}^{2}\right)\right.$; $i=1, \cdots, k\}$.

Lemma (4.8). Let $\mathscr{F}$ be as in Proposition (4.7). Then

$$
X_{s}^{\prime}\left(\tilde{\xi}_{I_{1}}^{2}, \cdots, \tilde{\xi}_{I_{t}}^{2}\right) \in T_{\phi(\xi)} V(|\mathscr{F}|) \quad \text { for } \quad s=1, \cdots, k .
$$

Let $\mathscr{I}>\mathcal{G}$ and let $\mathcal{G}=\left\{J_{1}, \cdots, J_{m}\right\}$. We can write $\mathscr{I}=\left\{J_{\nu^{\prime},} ; i=0, \cdots, m\right.$, $\left.j=1, \cdots, \nu_{i}\right\}$ so that $J_{i, j} \subset J_{i}$ where $J_{0}=\mathcal{G}^{c}$ by definition. Let $\bar{p}(u), \bar{q}(u), q, p(u)$, $q(u), \eta, T$ and $\underline{r}$ be as $\S 3$. We consider the equality $\bar{p}(u)-\bar{q}(u)=$ $\sum_{\imath=0}^{m} \sum_{j}^{\nu} \underline{i}_{1}\left|J_{i, j}\right|\left(X\left(\widetilde{p(u)_{J_{i}, j}^{2}}\right)-X\left(\widetilde{\left(q(u)_{J_{i}}^{2}\right.}\right)\right)$. Then using Lemma (4.8), we do the same argument as for the $A_{l+1}$-discriminant to obtain

THEOREM (4.9). $\overline{\mathcal{S}}$ is a regular stratification with the unique tangential limits property. Namely (i) $T$ is generated by $\left\{X^{(j)}\left(\tilde{\eta}_{J_{i}}^{2}\right) ; i=0, \cdots, m, j=1, \cdots, \nu_{i}\right\}$. (ii) (Regularity) $\gamma \in T$.

\section{$\S 5$. $D_{l+1}$-arrangement.}

Let $\mathscr{H}$ be the arrangement corresponding to the root system of type $D_{l+1}$ 
in $\boldsymbol{R}^{l+1}$. $\mathscr{H}$ has $2\left(\begin{array}{c}l+1 \\ 2\end{array}\right)$ hyperplanes $\left\{\xi_{i} \pm \xi_{j}=0\right\}$. The $D_{l+1}$-arrangement is a sub-arrangement of $B_{l+1}$-arrangement. The Weyl group $W$ is a semi-direct product of the symmetric group $S_{l+1}$ and $(\boldsymbol{Z} / 2 \boldsymbol{Z})^{l}$ and it is a subgroup of the Weyl group of $B_{l+1}$. We denote the $B_{l+1}$-arrangement by $\mathscr{H}(B)$, the Weyl group of $B_{l+1}$ by $W(B)$ and the stratification of $B_{l+1}$ by $\mathcal{S}(B)$ to distinguish with those of $D_{l+1}$. A basis of the invariant polynomial rings is given by $\left\{t_{1}, \cdots, t_{l}, t\right\}$ where $t_{2}$ is defined by (4.1) and $t=\xi_{1} \cdots \xi_{l+1}$ (Chapter 6, [1]). We use the basis $\mathscr{B}=\left\{\zeta_{1}, \cdots, \zeta_{l}, t\right\}$ where

$$
\zeta_{\imath}=\xi_{1}^{22}+\cdots+\xi_{l+1}^{22} \quad(i=1, \cdots, l) .
$$

The existence of $t$ makes the situation more difficult than the cases of $A_{l+1}$ or $B_{l+1}$-arrangements. In fact, the unique tangential limits property does not hold in general for $D_{l+1}$-arrangement. We define $\Phi: C^{l+1} \rightarrow C^{l+1} / W \cong C^{l+1}$ by $\xi \mapsto$ $\left(\zeta_{1}(\xi), \cdots, \zeta_{l}(\xi), t(\xi)\right)$ and let $\phi$ be the restriction to $|\mathscr{H}|$. In this case, the tangential map does not split into one variable functions. We have

$$
d \Phi_{\xi}\left(\frac{\partial}{\partial \xi_{i}}\right)=\left(2 \xi_{i} X^{\prime}\left(\xi_{i}^{2}\right), t(\xi) / \xi_{i}\right)=\xi_{i}\left(2 X^{\prime}\left(\xi_{i}^{2}\right), t(\xi) / \xi_{\imath}^{2}\right)
$$

under the canonical identification $T_{\Phi(\xi)} C^{l+1} \cong C^{l+1}$. In this section, we assume that $X(x)=\left(x, \cdots, x^{l}\right) \in \boldsymbol{C}^{l}$. Let $\mathcal{E}_{\imath}$ be as in $\S 4$ and let $\mathcal{E}_{1}^{\prime}=\left\{\mathscr{F}=\left\{I_{1}, \cdots, I_{k}\right\} \in \mathcal{E}_{1}\right.$; $M(\mathscr{F}) \subset|\mathscr{H}|\}$. Let $\mathcal{E}_{2}^{\prime}$ be the image of $\mathcal{E}_{1}^{\prime}$ in $\mathcal{E}_{2}$. Let $M(\mathscr{F})$ be as in $\S 4$ for $\mathscr{F} \in \mathcal{E}_{1}^{\prime}$. Let $\mathcal{S}=\left\{M(\mathscr{F}) ; \mathscr{F} \in \mathcal{E}_{1}^{\prime}\right\}$. Then $\mathcal{S}$ gives a regular stratification of $|\mathscr{H}|$ which is the restriction of the stratification of $|\mathscr{H}(B)|$ to $|\mathscr{H}|$. Here is a difference from the stratification of $B_{l+1}$ : Let $\xi, \xi^{\prime} \in M(\mathscr{I})$ such that $\xi_{i}^{2}=\xi_{i}^{\prime 2}$ for $i=1, \cdots$, $l+1$. Then $\xi$ and $\xi^{\prime}$ are in the same $W$-orbit if and only if $t(\xi)=t\left(\xi^{\prime}\right)$. (See $\S 4.8$ of Chapter $6,[1]$.) Thus if $\mathscr{F}^{c}=\emptyset, M(\mathscr{I})$ has two $W$-orbits of strata. (These strata are in the same $W(B)$-orbit.) In particular, $V(|\mathscr{F}|)$ is the disjoint union of two strata if $\mathscr{F}^{c}=\emptyset$. Understanding this difference, we use the same notations as $\S 4$ for the convenience's sake. Note that this stratification is finer than the stratification $\mathcal{S}_{m \imath n}$ which is defined in $\S 1$. The reason that we consider the stratification $\mathcal{S}$ rather than $\mathcal{S}_{m \imath n}$ is technical. $\mathcal{S}$ is compatible with the $W$-action as $W$ is a subgroup of $W(B)$ and $|\mathcal{H}|$ is $W(B)$-invariant. We use the same partial ordering $>$ in $\mathcal{E}_{\imath}^{\prime}$ as in $\mathcal{E}_{\imath}$ for $i=1,2$. Proposition (4.3) and Proposition (4.4) are still true. Now we consider the situation of Proposition (4.7) and Proposition (4.8). We first prepare a lemma. Let $Y(x)=x X^{\prime}(x)$, $Z(x)=X(x) / x=\left(1, x, \cdots, x^{l-1}\right)$ and $W(x)=(Z(x), t / x)$. We consider their derived functions $Y_{k}\left(x_{1}, \cdots, x_{k}\right), Z_{k}\left(x_{1}, \cdots, x_{k}\right)$ and $W_{k}\left(x_{1}, \cdots, x_{k}\right)$. See (2.13) for the definition.

LEMMA (5.3). We have the following recursive formulae. 
(i)

$$
\left\{\begin{array}{l}
Y_{k}\left(x_{1}, \cdots, x_{k}\right)=X_{k-1}^{\prime}\left(x_{1}, \cdots, x_{k-1}\right)+x_{k} X_{k}^{\prime}\left(x_{1}, \cdots, x_{k}\right) \\
X_{k}\left(x_{1}, \cdots, x_{k}\right)=Z_{k-1}\left(x_{1}, \cdots, x_{k-1}\right)+x_{k} Z_{k}\left(x_{1}, \cdots, x_{k}\right) \\
W_{k}\left(x_{1}, \cdots, x_{k}\right)=\left(Z_{k}\left(x_{1}, \cdots, x_{k}\right),(-1)^{k-1} t / x_{1} \cdots x_{k}\right) .
\end{array}\right.
$$

$$
\begin{aligned}
& \left(X_{k}\left(x_{1}, \cdots, x_{k}\right), 0\right)=W_{k-1}\left(x_{1}, \cdots, x_{k-1}\right)+x_{k} W_{k}\left(x_{1}, \cdots, x_{k}\right) . \\
& \left\{\begin{array}{l}
Y_{k}(x, \cdots, x)=X^{(k-1)}(x) /(k-2) !+x X^{(k)}(x) /(k-1) ! \\
Z_{k-1}(0, \cdots, 0)=X_{k}(0, \cdots, 0)=X^{(k-1)}(0) /(k-1) !
\end{array}\right.
\end{aligned}
$$

Proof. The assertions in (i) are immediate by the definition and the inductive argument. The assertion (ii) follows from (i). The assertion (iii) is a corollary of (i) and Lemma (2.15).

Proposition (5.4). Let $\mathscr{T}=\left\{I_{1}, \cdots, I_{k}\right\} \in \mathcal{E}_{1}^{\prime}$ and let $\xi \in M(\mathscr{F})$. Let $\tilde{\xi}$ be as in (4.6). Then

(0) The restriction $\phi: M(\mathscr{F}) \rightarrow V(|\mathscr{F}|)$ is a finite covering.

(i) $T_{\phi(\xi)} V(|\mathscr{I}|)$ has dimension $k$ and it is generated by

$$
\left\{\left(2 \tilde{\xi}_{I_{i}} X^{\prime}\left(\tilde{\xi}_{I_{i}}^{2}\right), t(\xi) / \tilde{\xi}_{I_{i}}\right), 1 \leqq i \leqq k\right\} .
$$

(ii) $\left(Y_{s}\left(\tilde{\xi}_{I_{1}}^{2}, \cdots, \tilde{\xi}_{I_{s}}^{2}\right), 0\right) \in T_{\phi(\xi)} V(|\mathscr{F}|)$ for $s=2, \cdots, k$.

(iii) $\left(2 X_{s}^{\prime}\left(\tilde{\xi}_{I_{1}}^{2}, \cdots, \tilde{\xi}_{I_{s}}^{2}\right),(-1)^{s-1} t(\xi) / \tilde{\xi}_{I_{1}}^{2} \cdots \tilde{\xi}_{I_{s}}^{2}\right) \in T_{\phi(\xi)} V(|\mathscr{I}|), s=1, \cdots, k$.

Proof. The assertions (0) and (i) are immediate from (5.2). For (ii), we consider the equality

$$
\begin{aligned}
\left(2 \tilde{\xi}_{I_{i}}^{2} X^{\prime}\left(\tilde{\xi}_{I_{i}}^{2}\right), t(\xi)\right)-\left(2 \tilde{\xi}_{I_{j}}^{2} X^{\prime}\left(\tilde{\xi}_{I_{j}}^{2}\right), t(\xi)\right) & =\left(2 Y\left(\tilde{\xi}_{I_{i}}^{2}\right), t(\xi)\right)-\left(2 Y\left(\tilde{\xi}_{I_{j}}^{2}\right), t(\xi)\right) \\
& =2\left(\tilde{\xi}_{I_{2}}^{2}-\tilde{\xi}_{I_{j}}^{2}\right)\left(Y_{2}\left(\tilde{\xi}_{I_{i}}^{2}, \tilde{\xi}_{I_{j}}^{2}\right), 0\right) \in T_{\phi(\xi)} V(|\mathscr{F}|) .
\end{aligned}
$$

As $\tilde{\xi}_{I_{2}}^{2} \neq \tilde{\xi}_{I_{1}}^{2}$ for $i \neq j$, this implies our assertion for the case $s=2$. The rest of the calculation is completely parallel to the case of $B_{l+1}$. For the assertion (iii) we note that $\left(2 X^{\prime}\left(\tilde{\xi}_{I_{i}}^{2}\right), t(\xi) / \tilde{\xi}_{I_{i}}^{2}\right) \in T_{\phi(\xi)} V(|\mathscr{F}|)$ by (i). Then we consider the derived functions of this expression. As the $s$-fold derived function of $t(\xi) / x$ is $(-1)^{8-1} t(\xi) / x_{1} \cdots x_{s}$ by ( $\mathrm{i}$ ) of Lemma (5.3), the assertion follows immediately.

Now we consider the limit of the tangent spaces and the regularity problem. Let $p(u), q(u), \bar{p}(u), \bar{q}(u), \mathscr{F}, \mathcal{G}, \eta, \gamma$ and $T$ be as in $\S 3$. Let $\mathscr{F}=\left\{J_{\imath, j} ; 0 \leqq i \leqq m\right.$, $\left.1 \leqq j \leqq \nu_{i}\right\}$ and $\mathcal{G}=\left\{J_{1}, \cdots, J_{m}\right\}\left(\mathscr{T}, \mathcal{G} \in \mathcal{E}_{1}^{\prime}\right)$. We assume, as in $\S 4, J_{2, j} \subset J_{2}$ for $0 \leqq i \leqq m$ where $J_{0}=\mathcal{G}^{c}$.

Case A. Assume that $\mathscr{F}^{c} \neq \emptyset$. This implies that $t(p(u)) / \widetilde{p(u)_{J_{2}, j}} \equiv 0$ for any $0 \leqq i \leqq m$ and $1 \leqq j \leqq \nu_{i}$. Thus the tangent space $T_{\bar{p}(u)} V(|\mathscr{F}|)$ is generated by

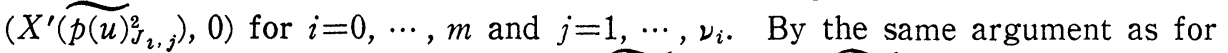

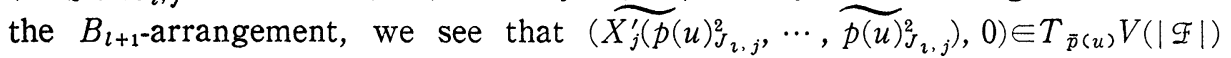
for $1 \leqq j \leqq \nu_{i}, 0 \leqq i \leqq m$. Taking the limit as $u \rightarrow 0$ and applying Lemma (2.15), we see that $\left(X^{(j)}\left(\tilde{\eta}_{J_{i}}^{2}\right), 0\right) \in T$ for $0 \leqq i \leqq m, 1 \leqq j \leqq \nu_{i}$. As $\operatorname{dim} M(\mathscr{F})=\sum_{l=1}^{m} \nu_{i} \leqq l$ 
and these vectors are linearly independent by the Generalized Vandermonde formula (Lemma (2.17)), $T$ is generated by $\left\{\left(X^{(j)}\left(\tilde{\eta}_{J_{i}}\right), 0\right) ; 0 \leqq i \leqq m, 1 \leqq j \leqq \nu_{i}\right\}$. Thus $T$ depends only on $q$ and $V(|\mathscr{F}|)$. By the expression

$$
\bar{p}(u)-\bar{q}(u)=\sum_{i=0}^{m} \sum_{j=1}^{\nu_{i}}\left|J_{i, j}\right|\left(X\left(\widetilde{p(u)_{J_{i}, j}^{2}}\right)-X\left(\widetilde{q(u)_{J_{i}}^{2}}\right), 0\right),
$$

the regularity also reduces to the case of $B_{l+1}$.

Case B. Assume that $\mathscr{F}^{c}=\emptyset$. We divide this case into two subcases.

Case B-1. Assume that $\mathcal{G}^{c} \neq \emptyset$ and $\mathscr{T}^{c}=\emptyset$.

This case is most complicated. Note that $t(p(u))= \pm \Pi_{\imath, j} \widetilde{p(u)_{J_{2, j}}^{\mid J_{\imath}}}$. We define $c \in C \cup\{\infty\}$ by

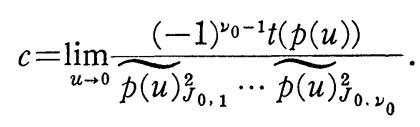

We claim that $T$ is generated by the following basis:

$$
\mathscr{B}_{c}=\left\{\left(X^{(\jmath)}\left(\tilde{\eta}_{J_{i}}^{2}\right), 0\right), 0 \leqq i \leqq m, 1 \leqq j \leqq \nu_{i},(i, j) \neq\left(0, \nu_{0}\right)\right\} \cup\left\{\left(\frac{2 X^{\left(\nu_{0}\right)}(0)}{\left(\nu_{0}-1\right) !}, c\right)\right\} .
$$

Recall that $\tilde{\eta}_{J_{2}}^{2}=\eta_{j}^{2}$ for any $j \in I_{\imath}$. In the case of $c=\infty$, the last vector in $\mathscr{B}_{c}$ is $e_{l+1}=(0, \cdots, 0,1)$ by definition. If $c \neq \infty$, the assertion is easily obtained by (iii) of Proposition (5.4) by taking the limit as $u \rightarrow 0$. Assume that $c=\infty$. By (iii) of Proposition (5.4), we can see that $T$ contains $e_{l+1}$ and $\left(X^{(j)}\left(\tilde{\eta}_{2}^{2}\right), 0\right)$ $\left(0<i \leqq m, 1 \leqq j \leqq \nu_{i}\right)$. On the other hand, we apply (ii) of Proposition (5.4) and (iii) of Lemma (5.3) to get $\left(X^{(j)}(0), 0\right) \in T$ for $j=1, \cdots, \nu_{0}-1$. As these vectors are linearly independent by the Generalized Vandermonde formula and $\operatorname{dim} M(\mathscr{I})$ $=\sum_{i=1}^{m} \nu_{i}, \mathscr{B}_{c}$ is a basis of $T$. Note that the last vector in $\mathscr{B}_{c}$ depends on the choice of $p(u)$ in general. See Remark (5.22) for detail. Now we consider the regularity. We start from the expression

$$
\begin{aligned}
\bar{p}(u)-\bar{q}(u)= & \sum_{i=1}^{m} \sum_{j=1}^{\nu_{i}}\left(\left|J_{i, j}\right|\left(X\left(\widetilde{p(u)_{J_{i}, j}^{2}}\right)-X\left(\widetilde{q(u)_{J_{i}}^{2}}\right), 0\right)\right. \\
& \left.+\sum_{j=1}^{\nu_{0}}\left|J_{0, j}\right| \widetilde{p(u)_{J_{0}, j}^{2}}\left(Z \widetilde{\left(p(u)_{J_{0, j}}^{2}\right.}\right), t(p(u)) /\left|J_{0}\right| \widetilde{p(u)_{J_{0, j}}^{2}}\right) .
\end{aligned}
$$

Using Lemma (5.3) and Proposition (2.14), we can rewrite (5.7) as

$$
\begin{aligned}
& \bar{p}(u)-\bar{q}(u)=\sum_{i=1}^{m} \sum_{j=1}^{\nu_{i}} \alpha_{\imath, j}(u)\left(X_{j+1}\left(\widetilde{q(u)_{J_{i}}^{2}}, \widetilde{p(u)_{J_{2}, 1}^{2}}, \cdots, \widetilde{p(u)_{J_{2}, j}^{2}}\right), 0\right)+ \\
& \left.\sum_{j=1}^{\nu_{0}} \alpha_{0, j}(u)\left(Z_{j} \widetilde{\left(\not(u)_{J_{0,1}}^{2}\right.}, \cdots, \widetilde{p(u)_{J_{0}, j}^{2}}\right),(-1)^{\jmath-1} t(p(u)) /\left|J_{0}\right| \prod_{h=1}^{J} \widetilde{p(u)_{J_{0}, h}^{2}}\right)
\end{aligned}
$$

where

$$
\left.\alpha_{0, j}(u)=\sum_{h=1}^{\nu_{0}}\left|J_{0, h}\right| \widetilde{p(u)_{J_{0}, h}^{2}} \prod_{s=1}^{j-1} \widetilde{\left(p(u)_{J_{0, h}}^{2}\right.}-\widetilde{p(u)_{J_{0, s}}^{2}}\right)
$$


(I) Assume first that $c \neq \infty, 0$. Let $\rho=\min \left\{\operatorname{order}\left(\alpha_{\imath, j}(u) ; 0 \leqq \imath \leqq m, 1 \leqq j \leqq \nu_{i}\right\}\right.$. Let $\alpha_{\imath, j}(u)=\alpha_{\imath, j} u^{\rho}+$ (higher terms). Applying Lemma (2.15) and Lemma (5.3), it is easy to see that

$$
\begin{aligned}
\lim _{u \rightarrow 0} \frac{\bar{p}(u)-\bar{q}(u)}{u^{\rho}}= & \sum_{i=1}^{m} \sum_{j=1}^{\nu_{i}} \alpha_{\imath, j}\left(X^{(j)}\left(\tilde{\eta}_{J_{i}}^{2}\right) / j !, 0\right) \\
& +\sum_{j=0}^{\nu_{0}-1} \alpha_{0, j}\left(X^{(j)}(0) / j !, 0\right)+\alpha_{0, \nu_{0}}\left(X^{\left(\nu_{0}\right)}(0) / \nu_{0} !, c /\left|J_{0}\right|\right) .
\end{aligned}
$$

Let $v$ be the vector defined by the right side of (5.10). As $v$ is a linear combination of the linearly independent vectors, $\gamma=[v]$. Here is a problem. The last vector $\left(X^{\left(\nu_{0}\right)}(0) / \nu_{0} !, c /\left|J_{0}\right|\right)$ in the linear combination of the right side of (5.10) is not necessarily contained in $T$. In fact, by (5.6) we have

$$
\left(X^{\left(\nu_{0}\right)}(0) /\left(\nu_{0}\right) !, c /\left|J_{0}\right|\right) \in T \Longleftrightarrow\left|J_{0}\right|=2 \nu_{0} .
$$

Thus we cannot conclude that $\gamma \in T$ so easily as before. The following assertion solves the problem.

Assertion (5.12). Assume that $\left|J_{0}\right| \neq 2 \nu_{0}$. Then $\alpha_{0, \nu_{0}}=0$.

Assuming this assertion for a while, we conclude from (5.10) that $\gamma \in T$ if $c \neq \infty, 0$.

Proof of (5.12). Assume that $\alpha_{0, \nu_{0}} \neq 0$. Let $\rho^{\prime}=\min \left\{\operatorname{order}\left(\widetilde{p(u)_{J_{0, j}}^{2}}\right) ; 1 \leqq j \leqq \nu_{0}\right\}$ and let $\widetilde{p(u)_{J_{0}, j}^{2}}=a_{j} u^{\rho^{\prime}}+$ (higher terms). Then by (5.9) we can write

$$
\alpha_{0, j}(u)=\sum_{h=1}^{\nu_{0}}\left|J_{0, h}\right| a_{h}\left(a_{h}-a_{\jmath-1}\right) \cdots\left(a_{h}-a_{1}\right) u^{\jmath \rho^{\prime}}+\text { (higher terms). }
$$

As $\operatorname{order}\left(\alpha_{0, \nu_{0}}(u)\right) \geqq \nu_{0} \rho^{\prime}$, the assumption that $\alpha_{0, \nu_{0}} \neq 0$ implies that $\rho \geqq \nu_{0} \rho^{\prime}$ and

$$
\sum_{n=1}^{\nu_{0}}\left|J_{0, h}\right| a_{h}\left(a_{h}-a_{\jmath-1}\right) \cdots\left(a_{h}-a_{1}\right)=0, \quad j=1, \cdots, \nu_{0}-1 .
$$

By an inductive argument on $j$, we can easily see that (5.13) is equivalent to the following:

$$
\sum_{h=1}^{\nu_{0}}\left|J_{0, h}\right| a_{h}^{j}=0, \quad j=1, \cdots, \nu_{0}-1 \text {. }
$$

Assume that $a_{j} \neq 0,1 \leqq j \leqq \nu_{0}$. This implies that $\operatorname{order}\left({\widetilde{p(u)_{J_{0}, j}}}\right)=\rho^{\prime} / 2$ for any $j$. Thus we have

$$
\operatorname{order}\left(t(p(u)) / \prod_{h=1}^{\nu_{0}} \widetilde{p(u)_{J_{0}, h}^{2}}\right)=\frac{\rho^{\prime}}{2}\left(\sum_{h=1}^{\nu_{0}}\left|J_{0, h}\right|-2 \nu_{0}\right)=\frac{\rho^{\prime}}{2}\left(\left|J_{0}\right|-2 \nu_{0}\right) .
$$

As we have assumed that $\left|J_{0}\right|-2 \nu_{0} \neq 0$, this implies that $c=0$ or $\infty$ as $J_{0}-2 \nu_{0}$ is positive or negative respectively. This is a contradiction to the assumption 
that $c \neq 0, \infty$. Thus by changing the ordering if necessary, we may assume that there exist $\beta\left(1 \leqq \beta<\nu_{0}\right)$ such that $a_{1}, \cdots, a_{\beta} \neq 0$ and $a_{\jmath}=0$ for $\beta<j \leqq \nu_{0}$. Thus (5.14) gives the following equlity

$$
\sum_{h=1}^{\beta} a_{h}^{\prime}\left|J_{0, h}\right|=0, \quad(j=1, \cdots, \beta) .
$$

This gives another contradiction to the following.

Lemma (5.17). Let $a_{1}, \cdots, a_{\beta}$ be given non-zero complex numbers. Then the equation

$$
\left(\begin{array}{ccc}
a_{1} & \cdots & a_{\beta} \\
a_{1}^{2} & \cdots & a_{\beta}^{2} \\
\vdots & \ddots & \vdots \\
a_{1}^{\beta} & \cdots & a_{\beta}^{\beta}
\end{array}\right)\left(\begin{array}{c}
n_{1} \\
\vdots \\
n_{\beta}
\end{array}\right)=\left(\begin{array}{c}
0 \\
\vdots \\
0
\end{array}\right) .
$$

has no strictly positive solutions. Here ${ }^{t}\left(n_{1}, \cdots, n_{\beta}\right)$ is strictly positive iff $n_{2}>0$, $i=1, \cdots, \beta$.

Assuming Lemma (5.17) for a moment, we can conclude that $\alpha_{0, \nu_{0}}=0$, completing the proof of Assertion (5.12) and the proof of the regularity in the case (I).

Proof of Lemma (5.17). Assume that ${ }^{t}\left(n_{1}, \cdots, n_{\beta}\right)$ is a strictly positive solution of $(*)$.

Step 1. Assume that $a_{\imath} \neq a$, if $i \neq j$. Then $(*)$ only has a trivial solution $n_{\imath}=0(i=1, \cdots, \beta)$ by the Vandermonde determinant formula.

Step. 2. In general, we consider the subdivision $\mathcal{K}=\left\{K_{1}, \cdots, K_{r}\right\}$ of $\{1, \cdots, \beta\}$ with the property that $a_{\imath}=a_{j} \Leftrightarrow \exists s ; i, j \in K_{s}$. Let $a_{K_{2}}=a$, for $j \in K_{\imath}$. Then $(*)$ implies that

$$
\left(\begin{array}{ccc}
a_{K_{1}} & \cdots & a_{K_{r}} \\
\vdots & \ddots & \vdots \\
a_{K_{1}}^{r} & \cdots & a_{K_{r}}^{r}
\end{array}\right)\left(\begin{array}{c}
m_{1} \\
\vdots \\
m_{r}
\end{array}\right)=\left(\begin{array}{c}
0 \\
\vdots \\
0
\end{array}\right)
$$

where $m_{\imath}=\sum_{j \in K_{i}} n_{\jmath}$. As $m_{2}>0$ by this expression, this gives a contradiction. by Step 1. This completes the proof of Lemma (5.17).

(II) Assume that $c=0$. Then $T$ is generated by $\left(X^{(j)}\left(\tilde{\eta}_{J_{i}}^{2}\right), 0\right)(0 \leqq i \leqq m$, $\left.1 \leqq j \leqq \nu_{i}\right)$. In particular, $T$ contains $\left(X^{\left(\nu_{0}\right)}(0), 0\right)$. We can see immediately from (5.10) that $\gamma \in T$.

(III) Assume that $c=\infty$. In this case, we know that $e_{l+1} \in T$. Let $\delta$ be the integer defined by

$$
\left.\left.\operatorname{order} \widetilde{\left(\not p(u)_{J_{0,1}}^{2}\right.} \cdots \widetilde{p(u)_{J_{0, \delta-1}}^{2}}\right)<\operatorname{order} t(p(u)) \leqq \operatorname{order} \widetilde{\left(p(u)_{J_{0,1}}^{2}\right.} \ldots \widetilde{p(u)_{J_{0, \delta}}^{2}}\right)
$$

We first rewrite the second term in (5.8) using (ii) of Lemma (5.3) as follows. 


$$
\begin{aligned}
& \left.\sum_{j=1}^{\nu_{0}} \alpha_{0, j}(u)\left(Z_{j} \widetilde{\left(p(u)_{J_{0,1}}^{2}\right.}, \cdots, \widetilde{\not p(u)_{J_{0,1}}^{2}}\right),(-1)^{\jmath-1} t(p .(u)) /\left|J_{0}\right| \prod_{h=1}^{j} \widetilde{p(u)_{J_{0, h}}^{2}}\right) \\
& \left.=\sum_{j=1}^{\delta-1} \alpha_{0, j}(u)\left(Z_{j} \widetilde{\left(\not p(u)_{J_{0,1}}^{2}\right.}, \cdots, \widetilde{p(u)_{J_{0, j}}^{2}}\right),(-1)^{\jmath-1} t(p(u)) /\left|J_{0}\right| \prod_{h=1}^{\jmath} \widetilde{p(u)_{J_{0, h}}^{2}}\right) \\
& \left.+\beta_{\delta}(u)\left(Z_{\delta} \widetilde{\left(\not p(u)_{J_{0,1}}^{2}\right.}, \cdots, \widetilde{p(u)_{J_{0}, \delta}^{2}}\right) b(u), 1\right) \\
& +\sum_{j=\delta+1}^{\nu_{0}} \beta_{j}(u)\left(X_{j}\left(\widetilde{p(u)_{J_{0,1}}^{2}}, \cdots, \widetilde{p(u)_{J_{0}, j}^{2}}\right), 0\right)
\end{aligned}
$$

where $b(u)=\left(\left|J_{0}\right| \Pi_{j=1}^{\delta} \widetilde{p(u}_{J_{0, j}}\right) /(-1)^{\delta-1} t(p(u))$ and $\left\{\beta_{j}(u)\right\}$ are rational functions. By the assumption, $\lim _{u \rightarrow 0}\left(Z_{\delta}\left(\widetilde{p(u)_{J_{0,1}}^{2}}, \cdots, \widetilde{p(u)_{J_{0}, \delta}^{2}}\right) b(u), 1\right)=\left(c^{\prime} X^{(\delta)}(0) / \delta !, 1\right)$ for some $c^{\prime} \in \boldsymbol{C}$. Let

$$
\begin{aligned}
& \rho_{1}=\min \left\{\operatorname{order}\left(\alpha_{i, j}(u) ; 1 \leqq i \leqq m, 1 \leqq j \leqq \nu_{i}, \text { or } i=0,1 \leqq j \leqq \delta-1\right\}\right. \\
& \rho_{2}=\min _{\delta \leqq j \leqq \nu_{0}} \operatorname{order}\left(\beta_{j}(u)\right), \quad \rho=\min \left\{\rho_{1}, \rho_{2}\right\} .
\end{aligned}
$$

Let $\alpha_{\imath, j}(u)=\alpha_{\imath, j} u^{\rho}+$ (higher terms) and $\beta_{j}(u)=\beta_{j} u^{\rho}+$ (higher terms). Then by (5.8) and (5.19), we obtain

$$
\begin{aligned}
\lim _{u \rightarrow 0} \frac{\bar{p}(u)-\bar{q}(u)}{u^{\rho}}= & \sum_{i=1}^{m} \sum_{j=1}^{\nu_{i}} \alpha_{\imath, j}\left(X^{(j)}\left(\tilde{\eta}_{J_{i}}^{2}\right) / j !, 0\right)+\sum_{j=1}^{\delta-1} \alpha_{0, j}^{\prime}\left(X^{(\jmath)}(0) / j !, 0\right) \\
& +\beta_{\delta}\left(c^{\prime} X^{(\delta)}(0) / \delta !, 1\right)+\sum_{j=\delta}^{\nu_{0}-1} \beta_{j+1}\left(X^{(j)}(0) / j !, 0\right) .
\end{aligned}
$$

By the Generalized Vandermonde formula, the right side of $(5.20)$ is non-zero. Thus we conclude by (5.6) that $\gamma \in T$.

Case B-2. Assume that $\mathscr{F}^{c}=\mathcal{G}^{c}=\emptyset$. First by (iii) of Proposition (5.4), $T$ contains the following vectors:

$$
\mathscr{B}=\left\{\left(\frac{2 X^{(j)}\left(\tilde{\eta}_{J_{i}}^{2}\right)}{(j-1) !}, \frac{(-1)^{j-1} t(\eta)}{\tilde{\eta}_{J_{2}}^{2 j}}\right), j=1, \cdots, \nu_{i}, i=1, \cdots, m\right\} .
$$

As they are linearly independent, $\mathscr{B}$ is a basis of $T$. Now we consider the regularity. Direct calculations seem to be complicated. We take the following viewpoints. We consider the mapping $\Psi: \boldsymbol{C}^{l+1} \rightarrow \boldsymbol{C}^{l+1},\left(\zeta_{1}, \cdots, \zeta_{l}, t\right) \mapsto\left(\zeta_{1}, \cdots, \zeta_{l}, t^{2}\right)$. As $\left\{\zeta_{1}, \cdots, \zeta_{l}, t_{l+1}\left(=t^{2}\right)\right\}$ is a basis of the invariant polynomial ring of the Weyl group $W(B)$ of the $B_{l+1}$-arrangement, we may consider the composition $\Psi \circ \Phi$ as the canonical quotient map of the $B_{l+1}$-discriminant. Note that $\Psi$ gives a double covering on $C^{l+1}-\left\{t_{l+1}=0\right\}$. For a given $\mathscr{I}=\mathcal{C}_{1}^{\prime}$, we denote by $V(|\mathscr{F}| ; B)$ the stratum of $|\mathscr{H}(B)| / W(B)$ which corresponds to $\mathscr{F}$. Then we have the commutative diagram. 


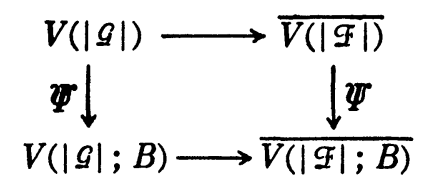

We have already shown the regularity for the pair $(V(|\mathscr{F}| ; B), V(|\mathcal{G}| ; B))$ in $\S 4$. Here we use the fact that the regularity does not depend on the choice of the coordinate functions ([7]). As $\Psi$ is locally a holomorphic diffeomorphism near $\bar{p}(0)=q$, the regularity for $(V|\mathscr{F}|), V(|\mathscr{G}|))$ follows immediately. Thus we have obtained

THEOREM (5.21). $\quad \overline{\mathcal{S}}$ is a regular stratification. The unique tangential limits property holds for the pair $(V(|\mathscr{I}|), V(|\mathcal{G}|))$ such that $\mathscr{I}^{c} \neq \emptyset$ or $\mathscr{F}^{c}=\mathcal{G}^{c}=\emptyset$.

Remark (5.22). Assume that $\mathscr{I}$ and $\mathcal{G}$ be as in Case B-1. We consider the possible values of $c$ in (5.5). If (i) $\left|J_{0, j}\right| \geqq 2$ for each $1 \leqq j \leqq \nu_{0}$, we can see that

$$
\begin{aligned}
c & = \pm \lim _{u \rightarrow 0}(-1)^{\nu_{0}-1} \prod_{i=1}^{m} \prod_{j=1}^{\nu_{i}} \widetilde{p(u)_{J_{l}, j}^{\left|J_{\imath}, j\right|}} \cdot \prod_{j=1}^{\nu_{0}} \widetilde{p(u)_{J_{0}, j}^{\left|J_{0, j}\right|-2}} \\
& = \begin{cases}0 & \text { if } \quad \exists j ;\left|J_{0, j}\right|>2 \\
\pm(-1)^{\nu_{0}-1} \prod_{l=1}^{m} \tilde{\eta}_{J_{l}}^{\left|J_{i}\right|} & \text { if } \quad \forall j ;\left|J_{0, j}\right|=2 .\end{cases}
\end{aligned}
$$

Thus in this case, the unique tangential property holds. Assume that (ii) $\left|J_{0, j}\right| \leqq 2$ for each $1 \leqq j \leqq \nu_{0}$ and $\left|J_{0, j}\right|=1$ for some $j$. Then $c=\infty$. Thus there is no problem for the unique tangential property. In particular, let $V(|\mathscr{F}|)$ be the stratum of the dimension $l$. We may assume that $\mathscr{I}=\{\{1,2\},\{3\}, \cdots$, $\{l+1\}\}$. Then the condition (ii) is always satisfied for any $V(|q|)$. Finally assume that (iii) there exist $a$ and $b$ such that $\left|J_{0, a}\right|=1$ and $\left|J_{0, b}\right|>2$. We claim that

ASSERTION. c can take any complex number or $\infty$.

Proof. We consider the curve $p(u)$ such that

(i) ${\widetilde{p(u)_{J_{0}, a}}}=u^{L},{\widetilde{p(u)_{J_{0, b}}}}=\varepsilon u^{M}+\delta u^{M+1}, \widetilde{p(u)_{J_{0}, j}}=u+a_{j} u^{2}, \quad j \neq a, b$

$$
p(u)_{k}=\widetilde{p(u)_{J_{0},}}, \quad \text { if } \quad k \in J_{0,3}
$$

(ii) $\widetilde{p(u)_{J_{\imath}, j}}=\tilde{\eta}_{J_{\imath}}+a_{\imath, j} u \quad 1 \leqq j \leqq \nu_{i}, 1 \leqq i \leqq m$ and $p(u)_{k}=\left(\eta_{k} / \eta_{m\left(J_{i}\right)} \widetilde{p(u)_{J_{i}, j}}\right.$ if $k \in J_{i, j}$

where $\delta, a$, and $a_{\imath, j}$ are suitable complex numbers and the integer $L$ and $M$ are so chosen that $\operatorname{order}\left(t(p(u))=\operatorname{order}\left(\Pi_{j=1}^{\nu_{0}} \widetilde{p(u)_{J_{0, j}}^{2}}\right)\right.$. Then it is easy to see that $c= \pm(-1)^{\nu_{0}-1} \varepsilon^{\left|J_{0}, b\right|-2} \times \Pi_{\imath=1}^{m} \tilde{\eta}_{J_{\imath}}^{\left|\tilde{\eta} J J_{i}\right|}$. Thus $c$ can take any complex number 
by moving $\varepsilon$. The case $c=\infty$ can be treated in a similar way by choosing the integers $L$ and $M$ which satisfy $\operatorname{order} t(p(u))<\operatorname{order}\left(\Pi_{j=1}^{\nu 0} \widetilde{p(u)_{J_{0}, j}^{2}}\right.$.

Though we have treated only $A_{l+1}, B_{l+1}$ and $D_{l+1}$-arrangements in this paper, it is highly expected that the regularity will hold for the other cases.

Acknowledgement. Professor C. T. C. Wall wrote the author that the regularity assertion for the other classes of the discriminant varieties also holds. In fact, his proof seems much general.

\section{REFERENCES}

[1] N. Bourbaki, "Groupes et Algèbres de Lie, Chapitres 4, 5 et 6", Hermann, Paris, 1968.

[2] E. BRIESKoRn, Sur les groupes de tresses, in "Séminaire Bourbaki 1971/72, Lecture Note in Math. 317", Springer, Berlin/Heidelberg/New York, 1973, pp. $21-44$.

[3] P. Deligne, Les immeubles des groupes de tresses généralisés, Invent. Math. 17 (1972), 273-302.

[4] S. LANG, "Algebra", Addison-Wesley, Amsterdam-London-Manila-Singapore-Sydney-Tokyo, 1965.

[5] J. MATHER, Stratifications and Mappings, in "Dynamical Systems," edited by Peixoto, 1973, pp. 195-232.

[6] P. ORLIK, Introduction to arrangements, preprint.

[7] H. Whitney, Tangents to analytic variety, Ann. Math. 81 (1964), 496-546.

Department of Mathematics

TOKyo Institute of TECHNOLOGy

Oh-oKayama, Meguro-ku, ToKyo 152

JAPAN 